International Journal of Biology, Pharmacy and Allied Seiences (IJBPAS) 'A Bridge Betuen Caboratory and QRendo'

www.iibpas.com

\title{
DESIGN AND OPTIMIZATION OF IBRUTINIB SOLID LIPID NANOPARTICLES USING DESIGN OF EXPERIMENT
}

\section{JARPULA ARUN KUMAR ${ }^{1}$, D. V. R. N. BHIKSHAPATHI ${ }^{2 *}$, PALANATI MAMATHA ${ }^{3}$}

1: Research Scholar, Career Point University, Kota, Rajasthan-325003, India

2: Research Supervisor, Career Point University, Kota, Rajasthan-325003, India

3: Teegala Ram Reddy College of Pharmacy, Pragathi Colony, Meerpet, Hyderabad-500097,

Telangana, India

*Corresponding Author: Dr. D. V. R. N. Bhikshapathi: E Mail: dbpathi71@gmail.com

Received 25 ${ }^{\text {th }}$ June 2021; Revised 28 ${ }^{\text {th }}$ July 2021; Accepted $29^{\text {th }}$ Aug. 2021; Available online 25 ${ }^{\text {th }}$ Sept. 2021

https://doi.org/10.31032/IJBPAS/2021/10.9.1056

ABSTRACT

In the present investigation, an attempt was made to design and formulate solid lipid nanoparticles of the anticancer drug, ibrutinib, to increase their bioavailability and overcome the challenges associated with the drug. To optimize the formulation variables and process variables of these SLNs, a statistically experimental design methodology was employed properly. After selecting the critical variables affecting particle size, encapsulation efficiency, and drug loading, the response surface methodology (RSM), followed by central composite design (CCD) was employed to optimize the level of these variables. From the preliminary studies, it was found that the drug to lipid ratio (A), concentration of Poloxamer 188 (B) and concentration of soya lecithin (C), had a significant effect on the particle size (Y1) and encapsulation efficiency(Y2) of SLNs.About 20 experimental runs carried out to evaluate the effect of factors on variables chosen. The optimized levels and predicted values of $\mathrm{Y} 1$, and $\mathrm{Y} 2$ were obtained by utilizing the design software. The optimised formulation as observed at 0.23:56.37:60 ratio (A:B:C) whose particle size and encapsulation efficiency ranged between $125.42-127.74 \mathrm{~nm}$ and 79.12 to 80.23 $\mathrm{mV}$. The PDI was ranging from 0.291 to 0.652 and zeta potential between $-21.7 \pm 1.22 \mathrm{mV}$ to - 
$25.3 \pm 1.78 \mathrm{mV}$. The optimized formulation was evaluated for various parameters like particle size analysis, polydispersity index, zeta potential, encapsulation efficiency, drug loading capacity, SEM analysis. The results indicate spherical shape of individual particles, confirming amorphisation of drug. The dissolution studies indicated maximum release of $95 \%$ within $24 \mathrm{~h}$. The optimized formulation found stable for 90 days.

\section{Keywords: Ibrutinib, B cell cancers, Central composite design (CCD), Solid lipid nanoparticles}

\section{(SLN), Particle size, Encapsulation efficiency}

\section{INTRODUCTION}

The SLNs are the alternative version of emulsions in which the liquid oil is replaced by solid lipids. Specific advantages include modulation of drug release, increased drug stability, exclusion of organic solvents from the manufacturing process, manufacturing scalability and industrial adaptability. SLN incorporate drugs into their lipid matrix to prevent drug efflux from P-glycoprotein, a multidrug efflux pump [1-5]. Compared with polymeric nanoparticles, SLN are more biocompatible and don't leave behind organic solvent residue in the final product. SLN also provide the feasibility of scale-up, hydrophilic and hydrophobic drug incorporation, sterilization and surface conjugation. However, some of the major drawbacks of SLNs are low drug loading, unpredictable drug release, and the risk of gelation due to polymorphism of the solid lipids [6, 7].

Ibrutinib is a small molecule drug that inhibits B-cell proliferation and survival by irreversibly binding the protein Bruton's tyrosine kinase. Blocking BTK inhibits the B-cell receptor pathway, which is often aberrantly active in B cell cancers. Ibrutinib displays an almost two-fold increase in its exposure when administered with food leads to decreased efficacy and safety of the drug. Due to poor solubility and hepatic first pass effects, it is commercially available in capsular dosage form with very high doses (140 mg per capsule), which results in severe gastrointestinal adverse effects. Thus, an improved oral formulation of ibrutinib is required with better bioavailability and higher efficacy [8-10]. In the present investigation, an attempt was made to design and formulate solid lipid nanoparticles of the anticancer drug, ibrutinib, to increase their bioavailability. The character of SLNs is changed by involved factors, depending on the preparation methods, and some of these factors are: type of lipid, type and concentration of emulsifier, and temperature 


\section{MATERIALS AND METHODS}

\section{Materials}

Ibrutinib was obtained as a gift sample from Dr. Reddy's laboratories Limited, Hyderabad, India. Glyceryl monostearate (GMS), glyceryl tripalmitate (tripalmitin), glyceryl tristearate (tristearin), Compritol 888 ATO (glyceryl behenate), glyceryl palmitostearate, dialysis tubing (molecular weight cut off $12-14 \mathrm{kDa}$ ), were obtained from Sigma Aldrich (St. Louis, MO, USA). Tween ${ }^{\circledR 80}$ was a product from SD Fine Chem Ltd (Mumbai, India). Soya Lecithin, Poloxamer-188 and PVA were gift samples from Dr. Roddy's laboratories 1td., India. All other chemicals and solvents were of analytical grade and were used without further purification.

\section{Development of SLN formulation \\ Preliminary experiments}

Various formulation parameters and process variables were optimized on the basis of their effect on particle size, polydispersity index, zeta potential and encapsulation efficiency. Formulations were prepared by changing one parameter at a time while keeping other parameters constant [11].

\section{Optimization of formulation parameters}

\section{Selection of excipients}

Nanoparticles were prepared using different lipids and were evaluated with respect to the particle size, PDI, and encapsulation efficiency. The particle size and PDI were determined using Malvern Zetasizer. Selection of the lipid, surfactant co-surfactant was made based on minimum particle size and PDI with maximum encapsulation efficiency [12].

The organic solvent is used to dissolve the lipids and chloroform was used in this study in varying volumes $(1-5 \mathrm{~mL})$. The formulation showing good particle size with minimum volume of solvent was selected.

\section{Optimization of process variables}

Various critical process variables which may have significant effect on the critical quality attributes were identified for each step involved in the formulation, that is, Homogenization speed and time, sonication time and stirring speed and time. Preliminary optimization of Homogenization speed and time, sonication time and stirring speed and time were carried out by conducting the experiments at four different RPM (4,000 to $8,000)$ for different time durations $(2,4,6$ and 8 minutes) at room temperature to get course emulsion. Then this course emulsion was sonicated for different time period (5-20 min) to get a nano-emulsion. Finally, formulation was stirred to evaporate the organic solvent and to get nanoparticles. The formulation was stirred at different speed 
(500, 1000 and $1500 \mathrm{rpm})$ and for different time period (1, 2 and $3 \mathrm{~h}$ ) for optimization [13].

\section{Drug-excipients compatibility study}

Compatibility study of drug with selected excipients was performed in order to assess the interaction between drug and polymer in physical state [14].

\section{Design of experiments (DoE)}

The central composite design (CCD) was used to optimize the formulation variables for the preparation of ibrutinib loaded solid lipid nanoparticles. From the preliminary studies, it was found that the drug to lipid ratio (A), Concentration of Poloxamer 188 (B) and Concentration of soyalecithin (C), had a significant effect on the particle size (Y1) and encapsulation efficiency (Y2) of
SLNs. The range of level of each independent variable was set according to the preliminary experiments and is listed in Table 1. On the basis of the central composite design model provided by StatEase Design Expert ${ }^{\circledR}$ software V8.0.1, 20 model experiments were randomly arranged. For all the experiments the homogenization speed (8000 rpm), homogenization time (6 $\mathrm{min})$, sonication time (10 min), stirring speed (1000 rpm) and stirring time (3 h) were kept constant. The experimental conditions of all model experiments are summarized in table 2. The experiments were conducted as for the design and the obtained responses for the dependent variables (Particle size -Y1 and Encapsulation efficiency - Y2) were presented in Table 2 [15].

Table 1: List of dependent and independent variables in central composite design

\begin{tabular}{|c|c|c|c|c|c|c|}
\hline \multicolumn{3}{|c|}{ Independent variables } & \multicolumn{4}{|c|}{ Levels } \\
\hline Variable & Name & Units & -1 & +1 & $-\alpha$ & $+\boldsymbol{\alpha}$ \\
\hline $\mathbf{A}$ & Drug to lipid ratio & - & 0.1 & 0.3 & & \\
\hline $\mathbf{B}$ & $\begin{array}{c}\text { Concentration of poloxamer } \\
188 \\
\end{array}$ & mg & 50 & 100 & & \\
\hline $\mathbf{C}$ & $\begin{array}{c}\text { Concentration of soya } \\
\text { lecithim }\end{array}$ & mg & 20 & 60 & & \\
\hline \multicolumn{3}{|c|}{ Dependent variable } & \multicolumn{4}{|c|}{ Goal } \\
\hline Y1 & Particle size & $\mathbf{n m}$ & \multicolumn{4}{|c|}{ Minimize } \\
\hline Y2 & Encapsulation efficiency & $\%$ & \multicolumn{4}{|c|}{ Maximize } \\
\hline
\end{tabular}

Table 2: Central composite design with observed responses

\begin{tabular}{|c|c|c|c|c|c|}
\hline Run & $\begin{array}{c}\text { Drug to } \\
\text { lipid ratio } \\
\text { (A) }\end{array}$ & $\begin{array}{c}\text { Conc. of } \\
\text { poloxamer } 188(B)\end{array}$ & $\begin{array}{c}\text { Conc. of soya } \\
\text { lecithin (C) }\end{array}$ & Particle size (Y1) & $\begin{array}{c}\text { Encapsulation } \\
\text { efficiency } \\
\text { (Y2) }\end{array}$ \\
\hline 1 & 0.2 & 75 & 40 & 138.72 & 83.56 \\
\hline 2 & 0.1 & 50 & 20 & 332.56 & 87.34 \\
\hline 3 & 0.2 & 75 & 40 & 138.35 & 82.82 \\
\hline 4 & 0.2 & 107.9019 & 40 & 172.34 & 86.56 \\
\hline 5 & 0.1 & 100 & 60 & 326.65 & 97.8 \\
\hline 6 & 0.2 & 75 & 13.67852 & 142.98 & 79.49 \\
\hline 7 & 0.2 & 42.09815 & 40 & 176.73 & 79.23 \\
\hline 8 & 0.2 & 75 & 40 & 139.12 & 83.12 \\
\hline 9 & 0.068393 & 75 & 40 & 385.92 & 96.156 \\
\hline
\end{tabular}




\begin{tabular}{|l|c|c|c|c|c|}
\hline 10 & 0.2 & 75 & 40 & 137.93 & 82.98 \\
\hline 11 & 0.1 & 50 & 60 & 329.12 & 92.98 \\
\hline 12 & $\mathbf{0 . 3 3 1 6 0 7}$ & 75 & 40 & 102.78 & 70.67 \\
\hline 13 & 0.1 & 100 & 20 & 328.86 & 92.89 \\
\hline 14 & 0.2 & 75 & 40 & 139.12 & 82.34 \\
\hline 15 & 0.3 & 50 & 20 & 118.46 & 67.98 \\
\hline 16 & 0.3 & 50 & 60 & 112.94 & 73.42 \\
\hline 17 & 0.3 & 100 & 60 & 108.82 & 80.08 \\
\hline 18 & 0.2 & 75 & 40 & 139.78 & 82.38 \\
\hline 19 & 0.2 & 75 & 66.32148 & 137.23 & 86.82 \\
\hline 20 & 0.3 & 100 & 20 & 116.18 & 73.88 \\
\hline
\end{tabular}

\section{Measurement of responses}

\section{Particle size}

The average particle size of prepared formulation was evaluated by dynamic light scattering method using Malvern particle size analyzer (Master sizer 2000, Malvern, UK) [16]

\section{Encapsulation efficiency [17]}

A fixed quantity of SLNs dispersion $(10 \mathrm{ml})$ was taken in a centrifuge tube and centrifuged at 10,000 rpm for $20 \mathrm{~min}$ at room temperature (Remi Instruments Pvt. Ltd, India), the lipid portion was isolated, and the absorbance of the drug in the supernatant was determined spectrophotometrically at $\lambda \max 260 \mathrm{~nm}$ (Shimadzu 1800, Japan). The percent encapsulation efficiency was calculated by using the following equation.

$$
E E(\%)=\frac{\text { Totaldrug }(\mathrm{mg})-\text { Freedrug }(\mathrm{mg})}{\text { Totaldrug }(\mathrm{mg})} \times 100
$$

\section{Data analysis}

Linear, quadratic and cubic models can be used to describe the relationship between dependent and independent variables. Numerous statistical parameters, comprising the $p$ value of the model, $p$ value of lack of fit, multiple regression coefficient $\left(\mathrm{R}^{2}\right)$, adjusted multiple regression coefficient (adjusted $\mathrm{R}^{2}$ ), coefficient of variation were considered to select a suitable fitting model. The terms with $\mathrm{p}$ value greater than 0.0005 ware considered as insignificant and were eliminated from the model. The independent variables which do not contribute to the regression equation will be deleted one at a time by backward elimination procedure. Three dimensional response surface plots shows the functional relationship between a selected dependent variable and two independent variables. Perturbation and contour plots also can be used to visualize the effect of independent variables on the response parameters [18].

\section{Optimization and confirmation experiments}

Numerical optimization using the desirability approach was employed to locate the optimal composition for the preparation of ibrutinib SLN and to obtain the desired response. An 
optimized composition was developed by setting constraints on the dependent and independent variables. Subsequently, three additional confirmation experiments with varied molar concentrations of both the ingredients were conducted to verify the validity of the statistical experimental strategies [19].

\section{Preparation of Ibrutinib loaded SLN}

Solid lipid nanoparticles were prepared by a single emulsification method combined with solvent evaporation technique as reported elsewhere [20]. Ibrutinib (100 mg), Trimyristin (Dynasan-114) and soya lecithin were dissolved in $3 \mathrm{~mL}$ of chloroform and poured in to $10 \mathrm{~mL}$ of $1.5 \% \mathrm{w} / \mathrm{v}$ of and poloxamer 188 solutions. The dispersion was homogenized at $8000 \mathrm{rpm}$ for $8 \mathrm{~min}$ and sonicated for $10 \mathrm{~min}$. The mixture kept for stirring at $1000 \mathrm{rpm}$ for $3 \mathrm{~h}$. The nanoparticle dispersion was centrifuged at $12000 \mathrm{rpm}$ for $45 \mathrm{~min}$. The settled nanoparticles were washed 3-4 times with milliQ water and lyophilized using trehalosedihydrate as cryoprotectant.

\section{Characterization of SLN [21]}

The formulated SLNs were evaluated for particle size, PDI, zeta potential and encapsulation efficiency.

\section{Morphology by scanning electron microscopy (SEM)}

The morphology of nanoparticles was studied by Scanning Electron Microscope (SEM, Hitachi, Tokyo, Japan).

\section{In-vitro drug release studies [22]}

The in vitro drug release study was performed using the USP apparatus II for 48 h. A fixed volume of the SLN suspensions was diluted with the simulated intestinal fluid (SIF, pH 6.8) at a ratio of $1: 9$ and stirred at $37^{\circ} \mathrm{C}$ with the speed of $50 \mathrm{rpm}$. At predetermined time points, $2 \mathrm{ml}$ of dissolution medium was removed and ultrafiltered as described above and analyzed by UV visible absorption measurement at 260 $\mathrm{nm}$. The removed volume was replaced with the same volume of a fresh $\operatorname{SIF}\left(37^{\circ} \mathrm{C}\right)$

\section{Stability studies [23]}

Stability of ibrutinib SLNs suspension in screw-capped glass vials was evaluated over a time period of 60 days. Six samples were divided into two groups and stored at $25^{\circ} \mathrm{C}$ and $4^{\circ} \mathrm{C}$. Drug leakage from nanoparticles and mean particle size of the samples were determined at the end of $1,7,15,30,45$, and 60 days.

\section{RESULTS AND DISCUSSION}

\section{Formulation development}

Based on preliminary studies the trimyristin (Dynasan114) was selected as the lipid, poloxamer 188 as surfactant and Soya 
lecithin as co-surfactant were chosen for the further studies.

At $3 \mathrm{ml}$ the selected solvent yielded maximum encapsulation efficiency. Hence, further experiments were conducted at same volume. The SLN formulations prepared by single emulsification-solvent evaporation method.

\section{Optimization of process variables}

From the results obtained in terms of particle size and PDI, it was observed that as the homogenization time and/or homogenization speed increased, the particle size decreased which may be attributed to increased force of deforming droplets at higher speed leading to smaller particles. It was also observed that with the increase in homogenization time PDI also increased which may be due to formation of foam and more aggregation in the formulation. Best results were obtained by stirring at 8,000 RPM for 6 minutes. Hence further investigations were done with homogenization/ stirring speed of 8000 RPM for $6 \mathrm{~min}$.Then this course emulsion was sonicated for different time period (5-20 min) to get a nano-emulsion. Finally, formulation was stirred to evaporate the organic solvent and to get nanoparticles. The formulation was stirred at different speed (500, 1000 and $1500 \mathrm{rpm})$ and for different time period (1, 2, and $4 \mathrm{~h}$ ) for optimization.

\section{Drug-excipients compatibility study}

IR spectra of the Ibrutinib, Dynasan-114, soya lecithin, Poloxamer 188 and physical mixture indicated the presence of major characteristic peaks as shown in Figure 1. The major characteristic peaks of Ibrutinib were observed at same wave numbers indicating the absence of any chemical interactions of the drug with the selected excipients. However, some additional peaks were observed with physical mixture, which could be due to the presence of functional groups of excipients.

DSC thermograms of pure drug, all the selected excipients used, and the physical mixture of drug and excipients were taken to confirm the identity of the drug and to detect the interaction of the drug with the excipients. As it is seen in Figure 2, a sharp symmetric endothermic peak was observed at $159.1268{ }^{\circ} \mathrm{C}$ for pure Ibrutinib which corresponds to its melting point. The DSC curve of physical mixture exhibits endothermic peaks of all the components. DSC thermogram of physical mixture illustrated the clear distinct endothermic peak of the drug indicative of the absence of any chemical interactions of the drug with the selected excipients.

\section{Design of experiment (DoE)}


Twenty experiments were required for the response surface methodology based on the Central Composite design. Based on the experimental design, the factor combinations yielded different responses as presented in Table 2. These results clearly specify that the dependent variables are strongly dependent on the selected independent variables as they show a wide variation among all the 20 batches. Data were analyzed using Stat-Ease Design Expert ${ }^{\circledR}$ software V8.0.1 to obtain analysis of variance (ANOVA), regression coefficients and regression equation. Mathematical relationships were generated using multiple linear regression analysis for the mentioned variables. These equations represent the quantitative effect of drug to lipid ratio (A), concentration of poloxamer 188(B) and concentration of soya lecithin (C) and their interaction on Particle size (Y1), and Encapsulation efficiency (Y2).

The values of the coefficients of $\mathrm{A}, \mathrm{B}$ and $\mathrm{C}$ are related to the effect of these variables on the responses Y1, and Y2. Coefficients with more than one factor term and those with higher order terms represent interaction terms and quadratic relationship respectively. A positive sign represents synergistic effect, while a negative sign indicates antagonistic effect. A backward elimination procedure was adopted to fit the data to the quadratic model. Both the polynomial equations were found to be statistically significant $(\mathrm{P}<0.01)$, as determined using ANOVA as per the provisions of Design Expert software.

\section{Particle size}

The particle size of the nanoparticles was found to be in the range of $102.78-385.92$ $\mathrm{nm}$ as shown in Table 2. The influence of the main and interactive effects of independent variables on the particle size was further elucidated using the perturbation, 3D response surface plots (Figure 3A, 3B \& 3C). These figure clearly shows that $\mathrm{A}$ has the main and the major effect on $\mathrm{Y} 1$ followed by $\mathrm{C} \& \mathrm{~B}$ which have moderate effect on Y1. At low levels of A (drug to lipid ratio), Y1 increases from $326.65 \mathrm{~nm}$ to $332.56 \mathrm{~nm}$. Similarly, at high levels of A, Y1 increases from $108.82 \mathrm{~nm}$ to $118.46 \mathrm{~nm}$. At low levels of B, Y1 decreases from $332.56 \mathrm{~nm}$ to 112.94 nm. Similarly, at high levels of $\mathrm{B}, \mathrm{Yi}$ decreases from $328.86 \mathrm{~nm}$ to $108.82 \mathrm{~nm}$. At low levels of C, Y1 decreases from 332.56 $\mathrm{nm}$ to $116.18 \mathrm{~nm}$. Similarly, at high levels of C, Y1 decreases from $329.12 \mathrm{~nm}$ to 108.82 $\mathrm{nm}$.

\section{Encapsulation efficiency}

Encapsulation efficiency of nanoparticles was found to be in the range of $67.98-97.8$ $\%$ as shown in Table 2. Results of the equation indicate that $\mathrm{A}$ has negative effect 
and $\mathrm{B} \& \mathrm{C}$ have positive effect on encapsulation efficiency. The perturbation and $3 \mathrm{D}$ response surface plots (Figure $\mathbf{4 A}$, 4B \& 4C). At low levels of A, Y2 decreased from97.8 \% to $87.34 \%$.Similarly, at high levels of A, Y2 decreased from $80.08 \%$ to $67.98 \%$. At low levels of B, Y2 increased from $67.98 \%$ to $92.98 \%$. Similarly, at high levels of B, Y2 increased from $73.88 \%$ to $97.8 \%$. At low levels of C, Y2 increased from $67.98 \%$ to $92.89 \%$. Similarly, at high levels of $\mathrm{C}, \mathrm{Y} 2$ increased from $73.42 \%$ to $97.8 \%$.

\section{Optimization and confirmation experiments}

A numerical optimization technique using the desirability approach was employed to prepare Ibrutinib nanoparticles with the desired responses. Constraints like minimizing the particle size in addition to maximizing the encapsulation efficiency were set as goals to locate the optimum settings of independent variables. By utilizing the design software, we got one solution for optimized formulation with a desirability value of 0.677 . The optimized levels and predicted values of Y1, and Y2 are shown in Table 3. To verify these values, three batches of nanoparticles were prepared according to the predicted levels of A, B and C. The predicted and observed values are shown in Table 3. Obtained Y1, and Y2 values were in a close agreement with the predicted values. This demonstrated the reliability of the optimization procedure in predicting the operating parameters for the preparation of Ibrutinib nanoparticles. All the three batches of obtained Ibrutinib nanoparticles were subjected to further characterization.

The mean size of all the formulations was ranging from $125.42 \pm 6.64 \mathrm{~nm}$ to $127.74 \pm$ $6.64 \mathrm{~nm}$ (The PDI was ranging from 0.291 to 0.652, indicating the wide range of size distribution. The zeta potential values of SLN formulations were found to be in between $21.7 \pm 1.22 \mathrm{mV}$ to $-25.3 \pm 1.78 \mathrm{mV}$. Total encapsulation efficiency of the nanoparticles formulations was determined and found to be ranging from $79.12 \pm 2.36 \%$ to $80.23 \pm 2.11$ $\%$ (Table 4).

\section{SEM analysis}

SEM analysis revealed the spherical shape of individual particles. The images in Figure 5 confirmed the nano size of the particles. SEM results were also in concurrence with DLS particle size measurements.

Figure 6 shows the in vitro release profile of ibrutinib from nanoformulation in SIF $(\mathrm{pH}$ 6.8). The amount of drug released from nanoformulation was significantly higher than that from the ibrutinib suspension. This was due to the fact that Ibrutinib was 
hydrophobic in nature and its solubility increases with nanoparticles, which resulted in the faster and enhanced release of Ibrutinib from nanoparticles.

\section{Stability studies}

Stability studies indicates that no significant difference $(\mathrm{p}<0.05)$ was found in encapsulation efficiency and particle size of optimized formulation stored at refrigerated conditions and at room temperature.

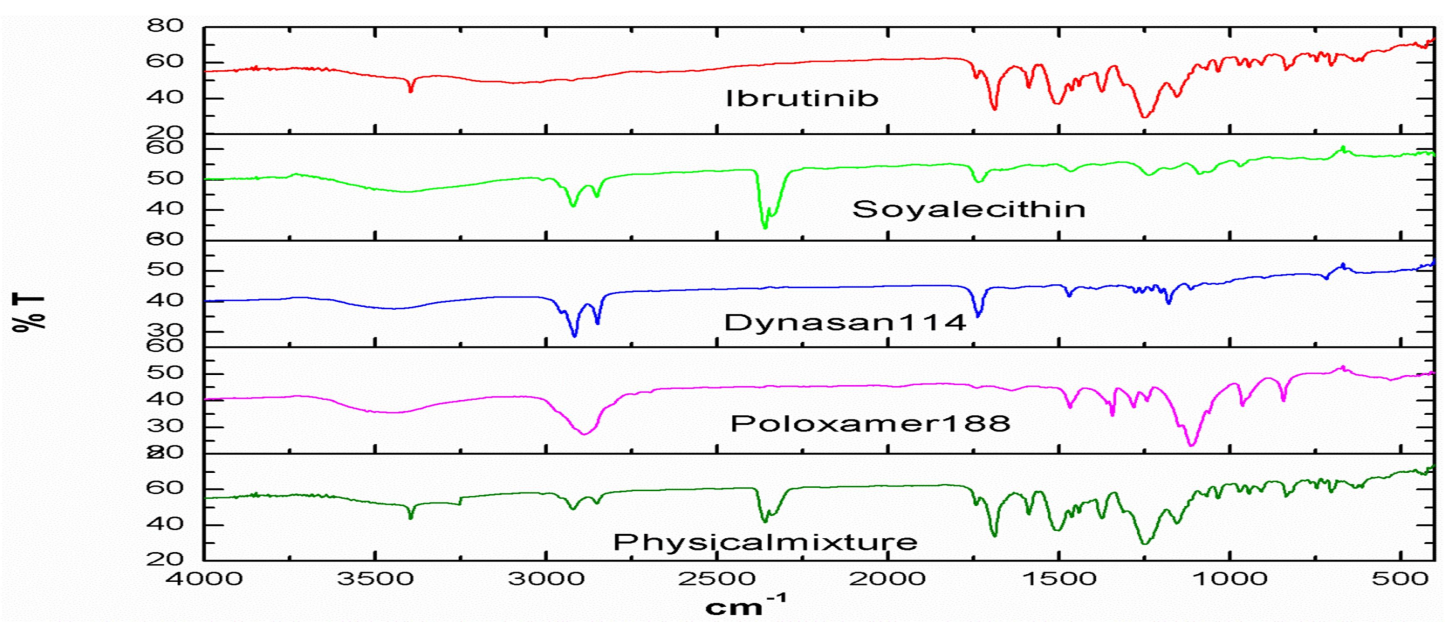

Figure 1: FTIR spectrum of Ibrutinib, Dynasan-114, Soya lecithin, Poloxamer 188 and physical mixture



Figure 2: DSC thermograms of Ibrutinib, Dynasan-114, Soya lecithin, Poloxamer 188 and physical mixture 

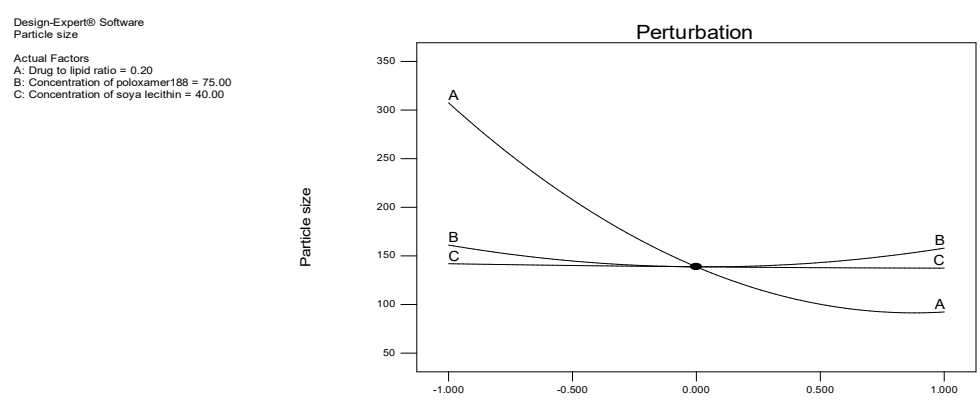

Deviation from Reference Point (Coded Units)

Figure 3A: Perturbation plot showing the effect of A, B and C on particle size
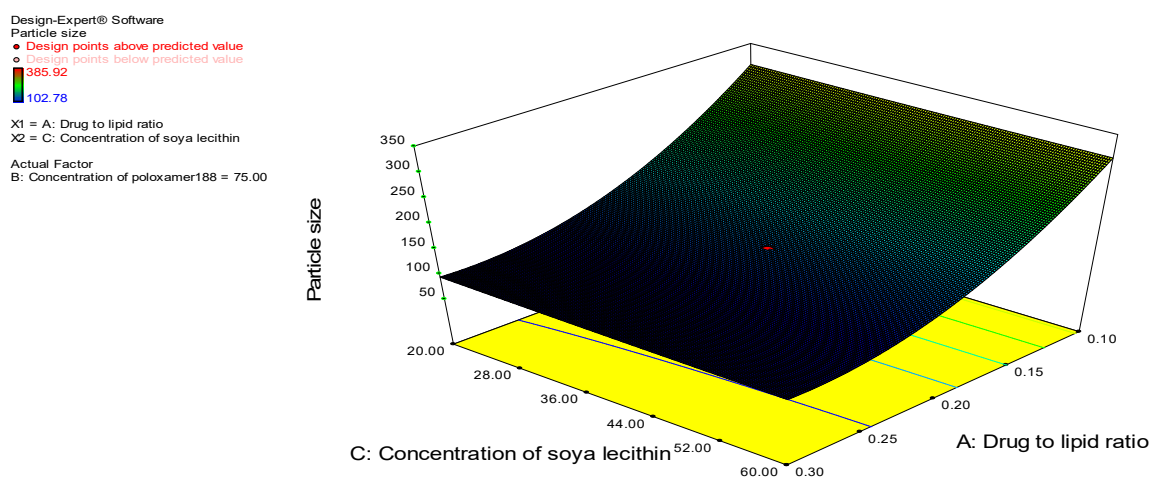

Figure 3B: Response surface plot showing the interactive effect of $A$ and $C$ at constant level of $B$
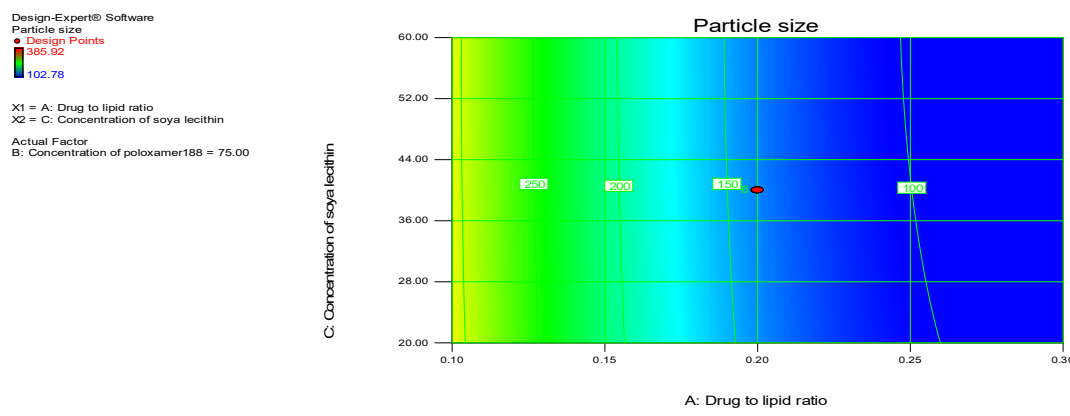

Figure 3C: Contour surface plot showing the interactive effect of $A$ and $C$ at constant level of $B$


Figure 4A: Perturbation plot showing the effect of A, B and C on \% encapsulation efficiency 


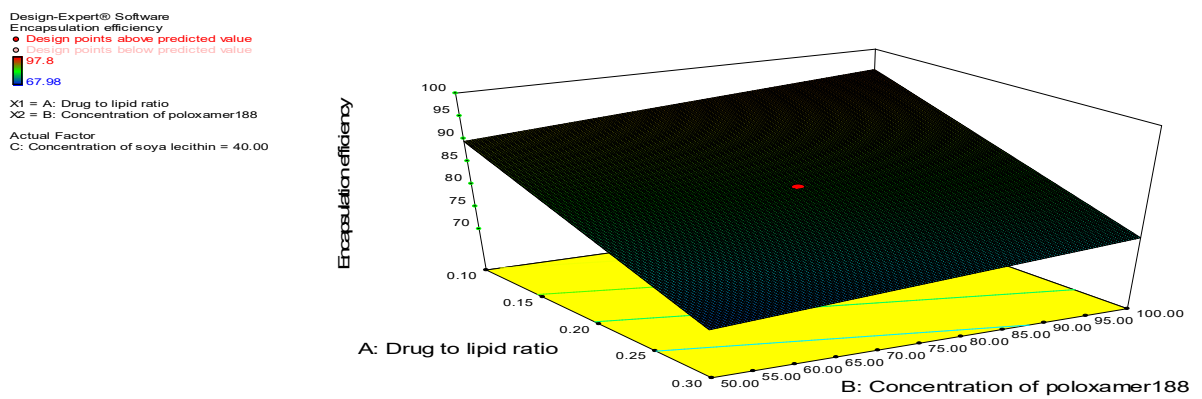

Figure 4B: 3D-Response surface plot showing the influence $A$ and $B$ on encapsulation efficiency at constant level of $C$

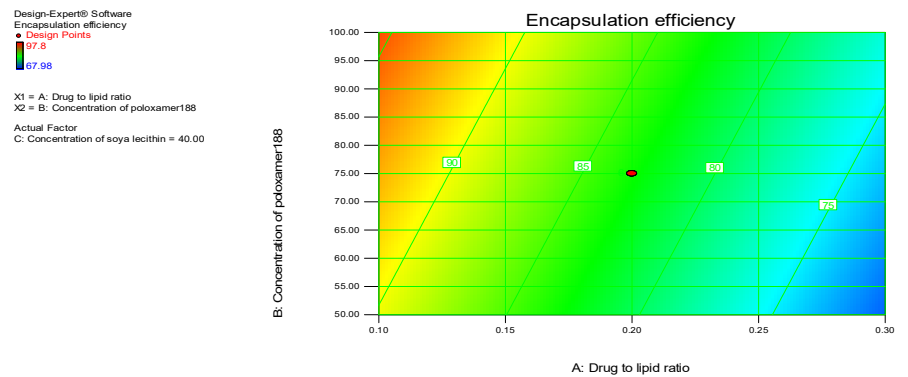

Figure 4C: Contour plot showing the influence $A$ and B on encapsulation efficiency at constant level of C

Table 3: Optimized values obtained by the constraints applies on Y1, and Y2

\begin{tabular}{|c|c|c|c|c|c|c|}
\hline \multirow[t]{2}{*}{ Independent variable } & \multirow{2}{*}{$\begin{array}{c}\text { Nominal } \\
\text { values }\end{array}$} & \multicolumn{2}{|c|}{ Predicted values } & \multicolumn{3}{|c|}{ Observed values } \\
\hline & & $\begin{array}{l}\text { Particle } \\
\text { size (Y1) }\end{array}$ & \begin{tabular}{|l|}
$\begin{array}{l}\text { Encapsulation } \\
\text { efficiency (Y2) }\end{array}$ \\
\end{tabular} & $\begin{array}{c}\text { Bat } \\
\text { ch }\end{array}$ & Particle size (Y1) & $\begin{array}{c}\text { Encapsulation } \\
\text { efficiency (Y2) }\end{array}$ \\
\hline \multirow{2}{*}{ Drug lipid ratio (A) } & \multirow[t]{2}{*}{0.23} & \multirow{4}{*}{123.199} & \multirow{4}{*}{80.94} & F1 & $126.53 \pm 7.26$ & $79.38 \pm 1.13$ \\
\hline & & & & F2 & $127.74 \pm 6.64$ & $80.23 \pm 2.11$ \\
\hline $\begin{array}{l}\text { Conc. of poloxamer } 188 \\
\text { (B) }\end{array}$ & 56.37 & & & F3 & $125.42 \pm 4.34$ & $79.12 \pm 2.34$ \\
\hline Conc. of soya lecithin (C) & 60 & & & & & \\
\hline
\end{tabular}

Table 4: The mean particle size, PDI, zeta potential and encapsulation efficiency of optimized formulations

\begin{tabular}{|c|c|c|c|c|}
\hline Batch & MPS \pm SD $(\mathbf{n m})$ & PDI & ZP \pm SD $(\mathbf{m V})$ & $\%$ EE \pm SD \\
\hline F1 & $\mathbf{1 2 6 . 5 3} \pm 7.26$ & $\mathbf{0 . 2 9 1}$ & $\mathbf{- 2 1 . 7} \pm 1.22$ & $\mathbf{7 9 . 3 8} \pm \mathbf{1 . 1 3}$ \\
\hline F2 & $\mathbf{1 2 7 . 7 4} \pm 6.64$ & $\mathbf{0 . 6 5 2}$ & $-24.7 \pm 2.33$ & $\mathbf{8 0 . 2 3} \pm \mathbf{2 . 1 1}$ \\
\hline F3 & $125.42 \pm 4.34$ & $\mathbf{0 . 4 9 6}$ & $-25.3 \pm 1.78$ & $\mathbf{7 9 . 1 2} \pm \mathbf{2 . 3 4}$ \\
\hline
\end{tabular}

$$
\mathrm{n}=\mathbf{3}(\mathbf{p}<\mathbf{0 . 0 5})
$$

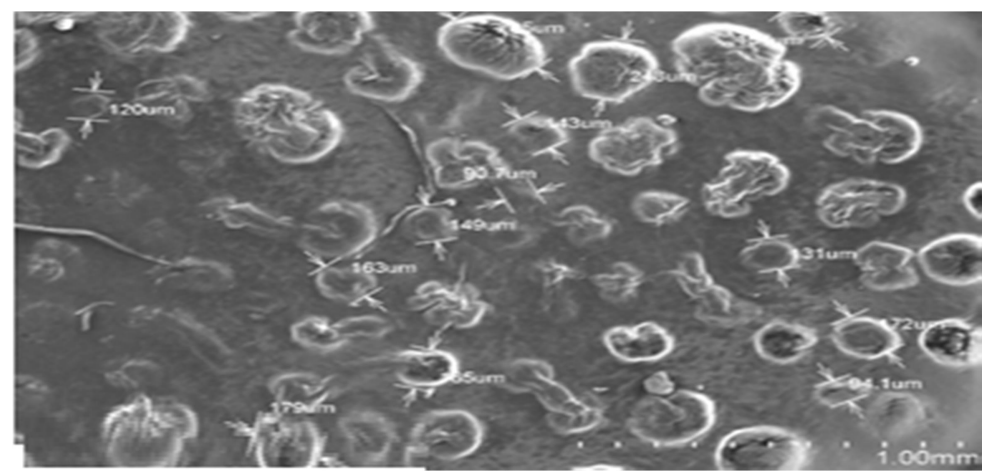

Figure 5: SEM image of Ibrutinib nanoparticles 


\section{CONCLUSION}

This work has demonstrated the use of a 3factor, 3-level Central composite design, regression analysis, 3-D response surface plots in optimizing the formulation variables in the preparation of Ibrutinib nanoparticles. The prepared SLNs were found to have an imperfect crystalline lattice and a spherical morphology. Significant enhancement of dissolution was observed with the nanoformulation of ibrutinib. This study suggests that solid lipid nanoparticles containing Ibrutinib could perform therapeutically better effects than the conventional formulations.

\section{REFERENCES}

[1] Markovic M, Ben-Shabat S, Aponick A, Zimmermann EM, Dahan A. Lipids and lipid-processing pathways in drug delivery and therapeutics. International journal of molecular sciences. 2020 Jan;21(9):3248.

[2] Chakraborty S, Shukla D, Mishra B, Singh S. Lipid-an emerging platform



for oral delivery of drugs with poor bioavailability. European Journal of Pharmaceutics and Biopharmaceutics. 2009 Sep 1;73(1):1-5.

[3] Mehnert W, Mäder K. Solid lipid nanoparticles: production, characterization and applications. Advanced drug delivery reviews. 2012 Dec 1;64:83-101.

[4] Bargoni A, Cavalli R, Zara GP, Fundarò A, Caputo O, Gasco MR. Transmucosal transport of tobramycin incorporated in solid lipid nanoparticles (SLN) after duodenal administration to rats. Part II-tissue distribution.

Pharmacological Research. 2001 May 1;43(5):497-502. [5] Cavalli R, Bargoni A, Podio V, Muntoni E, Zara GP, Gasco MR. Duodenal administration of solid lipid nanoparticles loaded with different percentages of tobramycin. Journal of pharmaceutical sciences. 2003 May 1;92(5):1085-94. 
[6] Rossi ED, Aínsa JA, Riccardi G. Role of mycobacterial efflux transporters in drug resistance: an unresolved question. FEMS microbiology reviews. 2006 Jan 1;30(1):36-52.

[7] Dingler A, Gohla S. Production of solid lipid nanoparticles (SLN): scaling up feasibilities. Journal of microencapsulation. 2002 Jan 1;19(1):11-6.

[8] Amin A, Ranjana HA. Bruton's tyrosine kinase inhibitors and their clinical potential in the treatment of B-cell malignancies: focus on ibrutinib. TherAdvHematol. 2014 Aug; 5(4): 121-133. doi: 10.1177/2040620714539906

[9] David MS, Brown JR. Ibrutinib: a first in class covalent inhibitor of Bruton's tyrosine kinase. Future Oncol. 2014 May; 10(6): 957-967. doi: $10.2217 /$ fon. 14.51

[10] Honigberg LA, Smith AM, Sirisawad M, Verner E, Loury D, Chang B, et al. The Bruton tyrosine kinase inhibitor PCI32765 blocks B-cell activation and is efficacious in models of autoimmune disease and B-cell malignancy. Proc Natl Acad Sci. 2010;107(29):13075-80.
[11] Hu FQ, Jiang SP, Du YZ, Yuan H, Ye YQ, Zeng S. Preparation and characteristics of monostearin nanostructured lipid carriers. International journal of pharmaceutics. 2006 May 11;314(1):83-9.

[12] Anton N, Benoit JP, Saulnier P. Design and production of nanoparticles formulated from nano-emulsion templates-a review. J Control Release 2008;128:18599.

[13] Nazzal S, Khan MA. Response surface methodology for the optimization of ubiquinone selfnanoemulsified drug delivery system. AAPS PharmSciTech. 2003;3:1-9. doi: 10.1208/pt030103.

[14] Anand Kumar Kushwaha, Parameswara Rao Vuddanda, Priyanka Karunanidhi, Sanjay Kumar Singh, Sanjay Singh, "Development and Evaluation of Solid Lipid Nanoparticles of Raloxifene Hydrochloride for Enhanced Bioavailability", BioMed Research International, vol. 2013, Article ID 584549, 9 pages, 2013.Doe

[15] S. Singh, A. K. Dobhal, A. Jain, J. K. Pandit, and S. Chakraborty, "Formulation and evaluation of 
solid lipid nanoparticles of a water soluble drug: zidovudine," Chemical and Pharmaceutical Bulletin, vol. 58, no. 5, pp. 650-655, 2010.

[16] V. V. Kumar, D. Chandrasekar, S. Ramakrishna, V. Kishan, Y. M. Rao, and P. V. Diwan, "Development and evaluation of nitrendipine loaded solid lipid nanoparticles: influence of wax and glyceride lipids on plasma pharmacokinetics," International Journal of Pharmaceutics, vol. 335, no. 1-2, pp. 167-175, 2007.

[17] Al-Tahami K. Preparation, characterization, and in vitro release of ketoprofen loaded alginate microspheres. Int J Appl Pharm 2014;6:9-12.

[18] Shivakumar HN, Patel PB, Desai BG, Ashok P, Arulmozhi S. Design and statistical optimization of glipizide loaded lipospheres using response surface methodology. Acta Pharm. 2007;57:269-285. doi: 10.2478/v10007-007-0022-8.

[19] Nazzal S, Khan MA. Response surface methodology for the optimization of ubiquinone selfnanoemulsified drug delivery system. AAPS PharmSciTech. 2003;3:1-9. doi: 10.1208/pt030103. [20] Pooja D, Kulhari H, Tunki L, Chinde S, Kuncha M, Grover P, Rachamalla SS, Sistla R. Nanomedicines for targeted delivery of etoposide to non-small cell lung cancer using transferrin functionalized nanoparticles. RSC advances. 2015;5(61):49122-31.

[21] Pn, R., and D. N. "Formulation, Development, And Characterisation Of Cilnidipine Loaded Solid Lipid Nanoparticles". Asian Journal of Pharmaceutical and Clinical Research, vol. 11, no. 9, Sept. 2018, pp. 120-5,

[22] Costa P, Lobo JM. Modeling and comparison of dissolution profiles. European journal of pharmaceutical sciences. 2001 May 1;13(2):12333.

[23] Zhang L, Pornpattananangkul D, Hu CM, Huang CM. Development of nanoparticles for antimicrobial drug delivery. Curr Med Chem 2010;17:585-90. 